\title{
Quantitative Analysis of Colistin-Resistant Escherichia coli in Retail Meat from Local Vietnamese Markets
}

\author{
Thang N. Nguyen, ${ }^{1}$ Diep T. Khong, ${ }^{1}$ Ha V. Le, ${ }^{1}$ Hoa T. Tran, ${ }^{1}$ Quang N. Phan, \\ Huong T. T. Le, ${ }^{1}$ Ryuji Kawahara $\mathbb{D}^{2},{ }^{2}$ and Yoshimasa Yamamoto $\mathbb{D D}^{3}$ \\ ${ }^{1}$ Center of Medical-Pharmaceutical Science and Technology Services, Thai Binh University of Medicine and Pharmacy, \\ Thai Binh, Vietnam \\ ${ }^{2}$ Department of Microbiology, Osaka Institute of Public Health, Osaka 537-0025, Japan \\ ${ }^{3}$ Life Science Research Center, Gifu University, Gifu 501-1194, Japan
}

Correspondence should be addressed to Yoshimasa Yamamoto; yyamamot@sahs.med.osaka-u.ac.jp

Received 7 December 2020; Revised 1 February 2021; Accepted 8 February 2021; Published 19 February 2021

Academic Editor: Maria Bayliak

Copyright (c) 2021 Thang N. Nguyen et al. This is an open access article distributed under the Creative Commons Attribution License, which permits unrestricted use, distribution, and reproduction in any medium, provided the original work is properly cited.

\begin{abstract}
The spread of drug-resistant bacteria via food has contributed to the dissemination of resistant bacteria among humans. However, the status of food contamination with resistant bacteria, particularly the quantitative level of resistant bacteria in food, has not yet been well elucidated. In this study, the abundance of colistin-resistant Escherichia coli in meat samples was quantified to understand the origin of the contamination of meat available in local Vietnamese markets. Fifteen samples each of chicken and pork meat purchased from local Vietnamese markets were assessed for the presence of colistin-resistant E. coli with the mobile colistin resistance gene, $m c r$. The results showed that $40 \%(6 / 15)$ and $66 \%(10 / 15)$ of the pork and chicken meat samples, respectively, were contaminated with colistin-resistant E. coli. The median quantitative levels of colistin-resistant $E$. coli in the contaminated pork and chicken samples were $1.8 \times 10^{4}$ and $4.2 \times 10^{3} \mathrm{CFU} / \mathrm{g}$, respectively. The results of phylogenetic analysis of isolates from a chicken meat sample showed that the contaminated colistin-resistant E. coli was a mix of multiple phylogenetical clones of bacteria that may have multiplied during sale. This is the first study to quantify the abundance of colistin-resistant $E$. coli in meat samples.
\end{abstract}

\section{Introduction}

The antibiotic colistin is used as the last resort for treating severe infections caused by multidrug-resistant Gramnegative bacteria, particularly carbapenem-resistant bacteria [1]. The recent emergence of acquired colistin resistance is considered a threat to global public health. Following the discovery of the first mobile colistin resistance gene, $m c r-1$, in 2015 [2], other $m c r$ variants $(m c r-2$ to $m c r-10)$ were also detected [3]. Thus, colistin resistance has disseminated worldwide, necessitating studies for monitoring and controlling the emergence of bacteria resistant to multiple drugs, including colistin.

Colistin has been widely used in animal husbandry, mostly orally, for various purposes [4-6]. In livestock, colistin may contribute to the emergence of colistin-resistant
(CR) bacteria due to selective pressure [4, 7]. Studies from Vietnam have shown that colistin and/or colistin-based drugs are commonly used in chicken and pig rearing $[6,7]$. The abuse of colistin in livestock may contribute to the introduction of CR bacteria. Supporting this hypothesis, Kawahara et al. [8] reported that in a rural area of Vietnam, approximately $100 \%$ chicken and pig fecal samples contained CR Escherichia coli with $m c r-1$ or $m c r-3$. A retrospective study on extended-spectrum $\beta$-lactamase- (ESBL-) producing E. coli strains isolated from healthy residents during 2013-2016 in the Thai Binh Province of Vietnam showed that $6.9 \%$ of the ESBL-producing E. coli strains possessed mcr-1 [9]. Another study from the same area conducted during 2017-2018 showed that approximately $70 \%$ of healthy residents carried CR E. coli with $m c r-1$ and/or $m c r-3$, and that $92.8 \%$ of those isolates were multidrug resistant [10]. Thus, 
the extremely high prevalence of CR E. coli in healthy residents might be attributed to the transmission of these bacteria from livestock to humans via diet. Increased attention should be paid toward monitoring CR bacteria and CRrelated genes in foods obtained from animal sources $[11,12]$.

As the presence of $E$. coli is indicative of food contamination, many studies have been conducted to detect the presence of CR E. coli in foods obtained from animals [13, 14]. However, these studies have only provided data on the rate of CR E. coli positivity and not on the abundance levels of CR E. coli in the samples. Here, we performed quantitative analysis of CR E. coli in chicken and pork meat samples for clarifying the status of the contamination of meat samples from local Vietnamese markets.

\section{Materials and Methods}

2.1. Sample Collection. Fifteen samples each of chicken and pork meat were purchased from 10 stores in two local markets in Thai Binh, Vietnam, from November 2018 to July 2019. For each meat type, only one sample was collected from a store. At some stores, multiple samples were collected at different times. The samples were placed in individual plastic bags and kept in a cooler box with ice until use. Bacterial isolates were cultured from the samples on the same day of collection.

2.2. Bacterial Culture. Twenty-five grams of each meat sample was placed in a stomacher bag containing $225 \mathrm{~mL}$ of buffered peptone water and hand-homogenized for $2 \mathrm{~min}$. The resulting supernatant was serially diluted (10-fold) using sterile saline. Next, $100 \mu \mathrm{L}$ of each diluted sample was inoculated onto both CHROMagar ECC (ECC) and CHROMagar COL-APSE (COL-APSE) (CHROMagar, Paris, France). ECC allows for the detection of E. coli-like and other coliform bacteria. COL-APSE is a selective medium for CR Gramnegative bacteria, often used to detect CR E. coli in food samples $[8,15]$. The number of $E$. coli-like colonies that could be distinguished based on colony color on both agar plates was determined after $24 \mathrm{~h}$ of incubation at $37^{\circ} \mathrm{C}$.

2.3. Selection of CR E. coli-Like Colonies. One representative E. coli-like colony grown on COL-APSE from each sample was isolated. In some samples, six randomly selected $E$. coli-like colonies from one COL-APSE agar plate were also isolated. These isolates were further characterized for bacterial identification, susceptibility to colistin, colistin resistance genes, and homology between isolates.

2.4. Characterization of the Isolates. The isolates were identified using biochemical tests with triple sugar iron slants (Japan BD, Tokyo, Japan), motility-indole-lysine medium (Japan BD), and cellobiose-lactose-indole- $\beta$-D-glucuronidase medium (Kyokuto Pharmaceutical, Tokyo, Japan).

Colistin susceptibility and minimum inhibitory concentrations (MICs) of the isolates were determined using the standard broth microdilution method [16]. MICs $\geq 4 \mu \mathrm{g} / \mathrm{mL}$ were interpreted as resistance to colistin.

The colistin resistance gene, $m c r$, was assessed using multiplex PCR. In brief, DNA was extracted from the isolates by boiling the bacterial suspension in tris(hydroxymethyl)aminomethane $(10 \mathrm{mM})$-ethylenediaminetetraacetic acid (1 mM) buffer pH 8.0 (Nippon Gene, Tokyo, Japan). The presence of $m c r-1, m c r-2, m c r-3, m c r-4$, and $m c r-5$ was detected using PCR, as described previously [11].

The genetic relatedness of isolates was assessed using pulsed-field gel electrophoresis (PFGE). XbaI-digested genomic DNA of isolates embedded in agarose was analyzed using the CHEF-DR III System (Bio-Rad, Hercules, CA, USA) according to a reported method [17].

\section{Results}

3.1. Detection of CR E. coli in Meat Samples. The contamination of meat samples with CR E. coli was assessed by culturing meat homogenate samples on COL-APSE. Representative E. coli-like colonies from each sample were isolated and identified using biochemical methods. All the samples contained E. coli, and resistance to colistin was also confirmed by assessing the MICs of the isolates. As shown in Table $1,40 \%$ and $66.7 \%$ of pork and chicken meat samples, respectively, contained CR E. coli.

3.2. Quantitative Contamination Level of Meat Samples with CR E. coli-Like Bacteria. All sample homogenates were serially diluted and cultured on both ECC and COL-APSE for quantifying the levels of E. coli-like and CR E. coli-like bacteria. The levels of CR E. coli-like bacteria varied across samples (Figure 1). The mean levels in chicken and pork meat were $2.8 \times 10^{4}$ and $5.7 \times 10^{4} \mathrm{CFU} / \mathrm{g}$, respectively, but the contamination frequency was lower in the latter (6/15 samples).

Because the number of $E$. coli-like colonies that grew on COL-APSE medium was high, none of the colonies were identified individually. Therefore, they were considered CR E. coli-like bacteria.

The level of E. coli-like bacteria in the samples was also determined by culturing the sample on ECC medium for comparing the CR bacteria levels. E. coli-like bacteria were detected in all CR-positive samples, except in chicken sample \#3 (Figure 1(b)). Conversely, not all samples positive for $E$. coli-like bacteria showed the presence of CR E. coli-like bacteria, such as three samples of nine E. coli-like bacteriapositive pork samples and four samples of $13 \mathrm{E}$. coli-like bacteria-positive chicken samples were negative for CRpositive E. coli-like bacteria. The quantitative levels of $E$. coli-like bacteria also varied across samples $\left(10^{2}-10^{7} \mathrm{CFU} / \mathrm{g}\right)$.

3.3. Characterization of CR E. coli Isolates. The homology among various $\mathrm{CR} E$. coli isolates derived from the same meat sample was assessed. Six randomly selected E. coli-like colonies grown on a COL-APSE plate by culturing a chicken sample were isolated, and colistin sensitivity and colistin resistance genes of these isolates were examined. The genetic relatedness of isolates confirmed to be CR E. coli possessing mcr- 1 was then assessed by PFGE analysis (Figure 2). The six isolates tested comprised four phylogenetically different clones, pulsotype 1 (TB0919C1.1 and TB0919C1.2), pulsotype 2 (TB0919C1.7), pulsotype 3 (TB0919C1.4 and TB0919C1.8), and pulsotype 4 (TB0919C1.5). 
TABLE 1: Detection of colistin-resistant (CR) Escherichia coli-like bacteria in food samples.

\begin{tabular}{|c|c|c|c|c|c|}
\hline & \multirow[t]{2}{*}{ No. of samples tested } & \multicolumn{2}{|c|}{$\begin{array}{c}\text { No. of samples } \\
\text { contaminated with CR } \\
\text { E. coli }\end{array}$} & \multicolumn{2}{|c|}{$\begin{array}{l}\text { Quantitative level of CR E. coli- } \\
\text { like bacteria in samples (CFU/g) }\end{array}$} \\
\hline & & $\#$ & $\%$ & Median & Range \\
\hline Pork & 15 & 6 & 40.0 & $1.8 \times 10^{4}$ & $10^{3}-10^{5}$ \\
\hline Chicken & 15 & 10 & 66.7 & $4.2 \times 10^{3}$ & $10^{2}-10^{5}$ \\
\hline
\end{tabular}

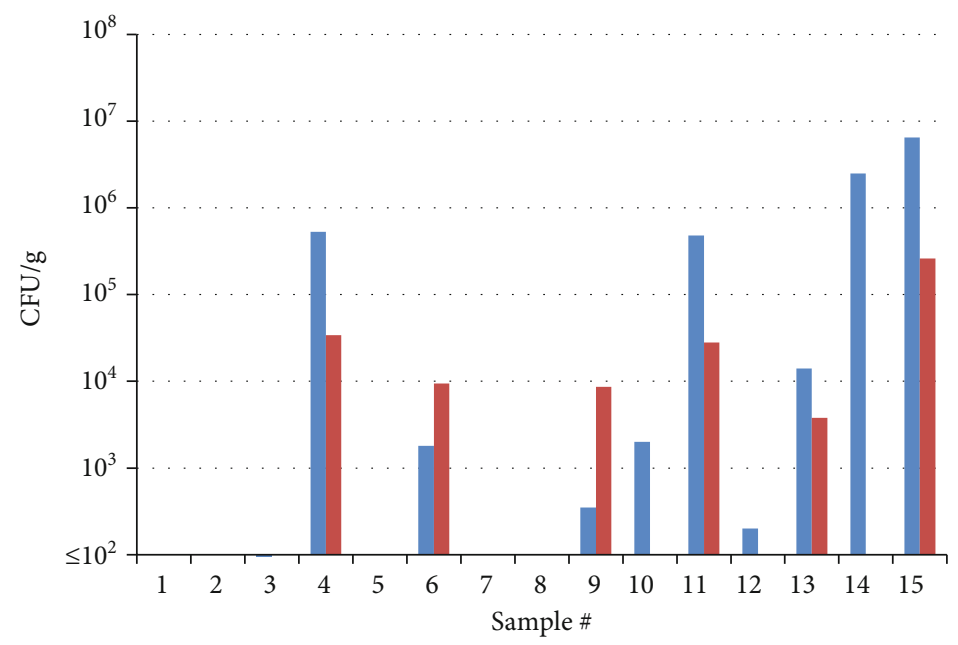

ECC

COL-APSE

(a)

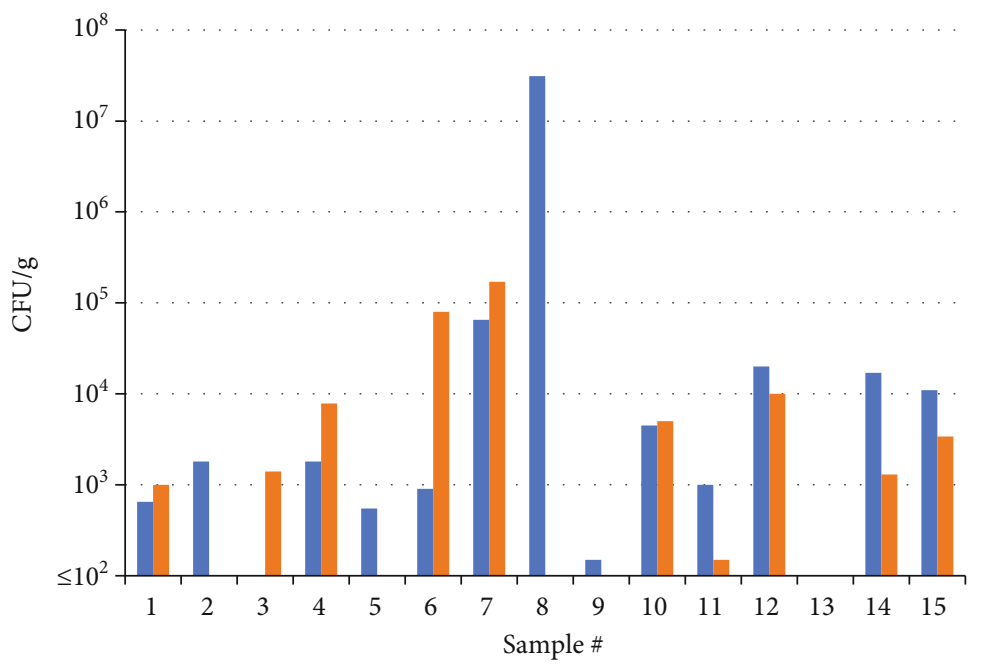

ECC

COL-APSE

(b)

Figure 1: Levels of Escherichia coli-like and colistin-resistant E. coli-like bacteria from individual samples. (a) Pork meat samples; (b) chicken meat samples. ECC indicates the level of E. coli-like bacteria. COL-APSE indicates the level of colistin-resistant (CR) E. coli-like bacteria. See Materials and Methods for the quantification of bacteria in samples. 

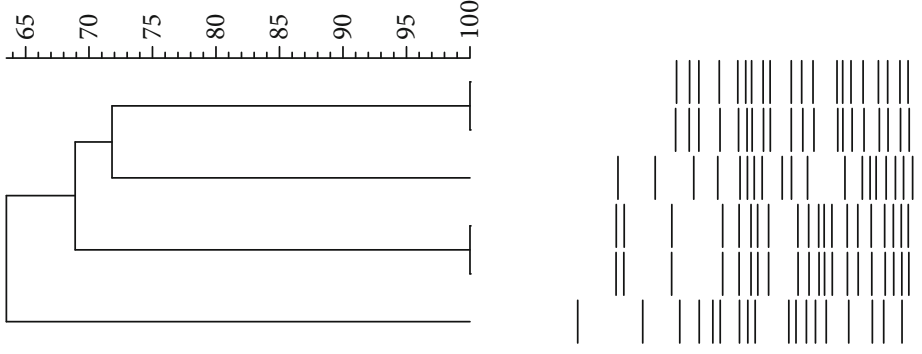

Strain

TB0919C1.1

ТВ0919C1.2

ТВ0919C1.7

TB0919C1.4

ТВ0919C1.8

ТВ0919С1.5

FIGURE 2: Dendrogram of pulsed-field gel patterns among colistin-resistant Escherichia coli colonies. A dendrogram of pulsed-field gel patterns among colistin-resistant Escherichia coli colonies on a CHROMagar COL-APSE agar plate cultured with a chicken meat sample homogenate was constructed. Strains from six randomly selected colonies from the plate were assessed.

\section{Discussion}

After $m c r-1$ was discovered in 2015, several retrospective studies showed that it is widely present in E. coli isolates from livestock, environment, food, and humans [2, 18-20]. However, the prevalence of $E$. coli possessing $m c r-1$ varies widely in foods of animal origin, depending on the sample type and location and time of sampling. For instance, a study showed that the prevalence of $m c r-1$-harboring E. coli in chicken meat samples collected from The Netherlands between 2009 and 2014 was 1.5\% [20], whereas another study on chicken meat samples collected from the same country in 2015 showed $24.8 \%$ prevalence [21]. Similarly, other studies have shown that the detection rate of $E$. coli possessing mcr-1 from chicken meat samples collected from Germany [19], China [2], Japan [22], Brazil [23], and Nepal [24] ranged from $1.4 \%$ to $19.5 \%$. More recently, a study in Tunisia showed that the rate of $E$. coli possessing $m c r-1$ from a chicken meat sample was relatively high (38.3\%) [25]. However, these detection rates were lower than those found in the present study (66.7\%). Likewise, highly variable results have been reported for pork meat samples. The detection rates of E. coli possessing $\mathrm{mcr}-1$ in pork meat samples collected from China [2] and Japan [14] were $19.0 \%$ and $3.1 \%$, respectively, whereas in Germany [19] and the Czech Republic [26], no pork meat sample showed contamination with $\mathrm{mcr}$-1-harboring E. coli.

The detection rate of CR E. coli from pork and chicken meat samples in this study was higher than that in previous studies; however, the rate is consistent with that in our previous studies from the same area, which showed a high detection rate of $m c r$-harboring $E$. coli in fecal samples from healthy humans and livestock (human: 69.39\%; chicken: 97.2\%; pig: $94.4 \%)[8,10]$. The magnitude of such variations in the detection rate might largely be because of the sampling conditions mentioned above; moreover, the primary culture medium used for CR bacterial growth seems to affect the results.

As most studies till date have focused merely on the detection rate of $m c r$-positive CR E. coli in foods, the quantitative level remains unclear. Because a higher density of bacteria in food indicates a higher risk in terms of food safety, even the contamination density may be mainly superficial; the quantitative level of CR E. coli in food is practically an important indicator of food safety.

Our findings reveal high mean levels of contaminated CR E. coli in chicken and pork samples. To our knowledge, this is the first study on the quantitative analysis of the contamination of meat samples with CR E. coli possessing mcr-1.

As E. coli colonies were distinguished from other colonies only on the basis of color, we recognized these as E. coli-like bacteria in quantitative experiments. However, the representative colonies grown on COL-APSE were isolated from all samples and identified as CR E. coli using a standard biochemical identification method, susceptibility tests to colistin, and detection of $m c r$ genes by PCR for the assessment of the contamination rate. The results showed that all these isolates were CR E. coli possessing mor-1. Therefore, the results of the quantitative experiments were highly likely to indicate the level of CR E. coli in food samples, although not all colonies were identified in the experiments. Other $m c r$ genes, such as $m c r-2$ to $m c r-5$, were not detected in the CR E. coli isolates tested. The results showing $m c r-1$ to be predominant are consistent with previous findings in livestock in the area [8].

Both ECC and COL-APSE can be used for quantifying the contamination levels in food samples. However, in some meat samples, the level of contamination with CR E. coli-like bacteria detected on COL-APSE was even higher than that with E. coli-like bacteria detected on ECC. This discrepancy might be caused by the presence of growth factor additives in COL-APSE, which is not disclosed by the manufacturer. Although it is not possible to determine the exact proportion of CR E. coli in the contaminating E. coli, with some exceptions, samples with a high number of contaminating $E$. coli might also have a high number of CR E. coli in food samples.

Generally, retail meats in local Vietnamese markets are processed as follows: shop owners in rural areas buy pigs and chickens, slaughter the animals themselves, and sell the meat before the day ends. The meat may get contaminated because of unhygienic slaughtering processes, the shopkeeper's hands, and flies or dirt present in the air during sale. Furthermore, because the meat is usually sold in hot and humid environments (average temperature during 
Vietnamese summers, $26-33^{\circ} \mathrm{C}$; average humidity, $>80 \%$ ) without hygienic storage facilities, the bacteria can grow easily after contaminating the meat. Considering all these factors, clone population analysis of contaminated CR E. coli in food is important for understanding the actual situation. The results of PFGE analysis of CR E. coli isolates revealed that six randomly selected isolates from the same chicken sample belonged to four phylogenetically different clones, implying that these strains can contaminate foods from different sources during various stages of food processing, such as slaughtering, preservation, or sale. In contrast, four of the six E. coli isolates were classified into two genetically related clones, indicating that the bacteria may have multiplied after contaminating the food.

The results obtained in this study are not absolute because they easily varied depending on the situation of food, but they are important as an indication of the reality of CR $E$. coli contamination in the local community. Thus, the study shows that meat in local markets is frequently contaminated with certain levels of CR E. coli. Such highly contaminated meats may contribute to the dissemination of CR bacteria in the community.

\section{Conclusions}

The study assessed the quantitative levels of CR E. coli in meat samples from local Vietnamese markets. The results showed that pork and chicken meat were frequently contaminated with CR E. coli at levels of $10^{4}$ and $10^{3} \mathrm{CFU} / \mathrm{g}$, respectively. Thus, the quantitative level of contamination with CR E. coli in retail meat has been clarified for the first time in this study.

\section{Data Availability}

The datasets used and/or analyzed during the current study are available from the corresponding author on reasonable request.

\section{Disclosure}

The preprint has been presented on the website https://www .researchsquare.com/article/rs-72382/v1.

\section{Conflicts of Interest}

The authors declare that there is no conflict of interest.

\section{Acknowledgments}

This work was supported by the Japan Society for the Promotion of Science KAKENHI (grant numbers 17H01687 and 20H00561).

\section{References}

[1] S. K. Kasiakou, A. Michalopoulos, E. S. Soteriades, G. Samonis, G. J. Sermaides, and M. E. Falagas, "Combination therapy with intravenous colistin for management of infections due to multidrug-resistant Gram-negative bacteria in patients with- out cystic fibrosis," Antimicrobial Agents and Chemotherapy, vol. 49, no. 8, pp. 3136-3146, 2005.

[2] Y. Y. Liu, Y. Wang, T. R. Walsh et al., "Emergence of plasmidmediated colistin resistance mechanism MCR-1 in animals and human beings in China: a microbiological and molecular biological study," The Lancet Infectious Diseases, vol. 16, no. 2, pp. 161-168, 2016.

[3] Y. Shen, R. Zhang, S. Schwarz et al., "Farm animals and aquaculture: significant reservoirs of mobile colistin resistance genes," Environmental Microbiology, vol. 22, no. 7, pp. 24692484, 2020.

[4] B. Catry, M. Cavaleri, K. Baptiste et al., "Use of colistincontaining products within the European Union and European economic area (EU/EEA): development of resistance in animals and possible impact on human and animal health," International Journal of Antimicrobial Agents, vol. 46, no. 3, pp. 297-306, 2015.

[5] T. Nakayama, M. Jinnai, R. Kawahara et al., "Frequent use of colistin-based drug treatment to eliminate extendedspectrum beta-lactamase-producing Escherichia coli in backyard chicken farms in Thai Binh Province, Vietnam," Tropical Animal Health and Production, vol. 49, no. 1, pp. 31-37, 2017.

[6] N. Van Cuong, N. T. Nhung, N. H. Nghia et al., "Antimicrobial consumption in medicated feeds in Vietnamese pig and poultry production," EcoHealth, vol. 13, no. 3, pp. 490-498, 2016.

[7] N. T. Nguyen, H. M. Nguyen, C. V. Nguyen et al., "Use of colistin and other critical antimicrobials on pig and chicken farms in southern Vietnam and its association with resistance in commensal Escherichia coli bacteria," Applied and Environmental Microbiology, vol. 82, no. 13, pp. 3727-3735, 2016.

[8] R. Kawahara, Y. Fujiya, T. Yamaguchi et al., "Most domestic livestock possess colistin-resistant commensal Escherichia coli harboring $\mathrm{mcr}$ in a rural community in Vietnam," Antimicrobial Agents and Chemotherapy, vol. 63, article e00594, 2019.

[9] R. Kawahara, D. T. Khong, H. V. Le et al., "Prevalence of $m c r-1$ among cefotaxime-resistant commensal Escherichia coli in residents of Vietnam," Infection and Drug Resistance, vol. Volume 12, pp. 3317-3325, 2019.

[10] Y. Yamamoto, R. Kawahara, Y. Fujiya et al., "Wide dissemination of colistin-resistantEscherichia coliwith the mobile resistance genemcrin healthy residents in Vietnam," Journal of Antimicrobial Chemotherapy, vol. 74, no. 2, pp. 523-524, 2019.

[11] T. Yamaguchi, R. Kawahara, K. Harada et al., "The presence of colistin resistance gene $m c r-1$ and -3 in ESBL producing Escherichia coli isolated from food in Ho Chi Minh City, Vietnam," FEMS Microbiology Letters, vol. 365, article fny100, 2018.

[12] F. Perez-Rodriguez and B. Mercanoglu Taban, "A state-of-art review on multi-drug resistant pathogens in foods of animal origin: risk factors and mitigation strategies," Frontiers in Microbiology, vol. 10, p. 2091, 2019.

[13] A. Ghafur, C. Shankar, P. GnanaSoundari et al., "Detection of chromosomal and plasmid-mediated mechanisms of colistin resistance in _Escherichia coli_and_Klebsiella pneumoniae from Indian food samples," Journal of Global Antimicrobial Resistance, vol. 16, pp. 48-52, 2019.

[14] Y. Nishino, Y. Shimojima, Y. Suzuki et al., "Detection of the mcr-1 gene in colistin-resistant Escherichia coli from retail meat in Japan," Microbiology and Immunology, vol. 61, no. 12, pp. 554-557, 2017.

[15] Y. Yamamoto, M. Calvopina, R. Izurieta et al., "Colistin-resistant Escherichia coli with $\mathrm{mcr}$ genes in the livestock of rural 
small-scale farms in Ecuador," BMC Research Notes, vol. 12, no. 1, pp. 121-126, 2019.

[16] M. A. Wikler, "Methods for dilution antimicrobial susceptibility tests for bacteria that grow aerobically: approved standard," CLSI (NCCLS), vol. 26, 2006M7-A7.

[17] E. M. Ribot, M. A. Fair, R. Gautom et al., "Standardization of pulsed-field gel electrophoresis protocols for the subtyping of Escherichia coli O157:H7, Salmonella, and Shigella for PulseNet," Foodborne Pathogens and Disease, vol. 3, no. 1, pp. 5967, 2006.

[18] Z. Shen, Y. Wang, Y. Shen, J. Shen, and C. Wu, "Early emergence of $m c r-1$ in Escherichia coli from food-producing animals," The Lancet Infectious Diseases, vol. 16, no. 3, p. 293, 2016.

[19] A. Irrgang, N. Roschanski, B. A. Tenhagen et al., "Prevalence of $\mathrm{mcr}-1$ in $E$. coli from livestock and food in Germany, 2010-2015," PLoS One, vol. 11, no. 7, article e0159863, 2016.

[20] M. F. Kluytmans-van den Bergh, P. Huizinga, M. J. Bonten et al., "Presence of $m c r-1$-positive Enterobacteriaceae in retail chicken meat but not in humans in the Netherlands since 2009," Euro Surveillance, vol. 21, no. 9, article 30149, 2016.

[21] E. J. A. Schrauwen, P. Huizinga, N. van Spreuwel, C. Verhulst, M. F. Q. Kluytmans-van den Bergh, and J. Kluytmans, "High prevalence of the $m c r-1$ gene in retail chicken meat in the Netherlands in 2015," Antimicrobial Resistance and Infection Control, vol. 6, no. 1, p. 83, 2017.

[22] Y. Ohsaki, W. Hayashi, S. Saito et al., "First detection of an Escherichia coli strain harboring the $m c r-1$ gene in retail domestic chicken meat in Japan," Japanese Journal of Infectious Diseases, vol. 70, no. 5, pp. 590-592, 2017.

[23] D. F. Monte, A. Mem, M. R. Fernandes et al., "Chicken meat as a reservoir of colistin-resistant Escherichia coli strains carrying mcr-1 genes in South America," Antimicrobial Agents and Chemotherapy, vol. 61, no. 5, 2017.

[24] P. R. Joshi, R. Thummeepak, U. Leungtongkam et al., "The emergence of colistin-resistant Escherichia coli in chicken meats in Nepal," FEMS Microbiology Letters, vol. 366, no. 20, 2019.

[25] B. Hassen, M. S. Abbassi, L. Ruiz-Ripa et al., "High prevalence of $\mathrm{mcr}-1$ encoding colistin resistance and first identification of bla $_{\mathrm{CTX}-\mathrm{M}-55}$ in ESBL/CMY-2-producing Escherichia coli isolated from chicken faeces and retail meat in Tunisia," International Journal of Food Microbiology, vol. 318, article 108478, 2020.

[26] T. Gelbicova, A. Barakova, M. Florianova et al., "Dissemination and comparison of genetic determinants of $m c r$-mediated colistin resistance in Enterobacteriaceae via retailed raw meat products," Frontiers in Microbiology, vol. 10, p. 2824, 2019. 